\title{
TECNOLOGIAS DE COMPUTAÇÃO GRÁFICA E APRENDIZAGEM VIRTUAL PARA VIABILIZAR PRÁTICAS EM DESENHO PARA ENGENHARIA DE FORMA REMOTA
}

Ludwig Bezerra Sales - ludwigbsales@alu.ufc.br

Helano Fontenele Alexandrino - helanofontenele@alu.ufc.br

Marcos Augusto Pereira Albuquerque Lima - marcospl@alu.ufc.br

Universidade Federal do Ceará, Curso de Engenharia de Computação

Campus do Pici, Bloco 725, Pici

60455-970 - Fortaleza - Ceará

Cely Martins Santos De Alencar - celyms@ufc.br

Universidade Federal do Ceará, Departamento de Integração Acadêmica e Tecnológica

Ac. Público, 710, Pici

60355-636 - Fortaleza - Ceará

Resumo: $O$ presente artigo mostra o relato da aprendizagem de forma remota na disciplina de Desenho para Engenharia (DPE) da Universidade Federal do Ceará, pelos alunos de Engenharia de Computação. Com o isolamento social causado pelo Covid-19, houve a necessidade de realizar o Ensino e as atividades remotamente. Assim, metodologia realizada foi o uso de tecnologias de web conferência para as reuniões e computação gráfica na construção dos desenhos. Neste relato de caso é descrito todos os passos executados pelos alunos, mediados pelas tecnologias de computação gráfica na modelagem de peças com técnicas do Desenho Geométrico. O sólido executado foi Playstation 4 (PS4) devido à presença de tal objeto no cotidiano dos integrantes do grupo. Os resultados obtidos foram satisfatórios e as experiências adquiridas, como responsabilidade individual, trabalho em grupo e colaboração, foram observadas nas competências avaliadas.

Palavras-chave: Aprendizagem, Ensino remoto, Desenho para Engenharia, Computação gráfica.

\section{INTRODUÇÃO}

A disciplina de Desenho, fundamental no primeiro ano das Engenharias, é composta por conteúdos, métodos e normativas fundamentais ao desenvolvimento e interpretação dos projetos, conceitos e ideais desenvolvidos nas Engenharias. Com as Tecnologias de 
aprendizagem e a computação gráfica atuais, o processo de produção das representações gráficas e o Ensino e aprendizado passa por grandes modificações.

As perceptíveis mudanças nos meios de comunicação ocorridas nas últimas duas décadas abrem espaço para novas formas de interação como relatado por Monnerat (2012). Assim, estas tecnologias associadas a metodologias de aprendizagem colaborativa já existentes, podem funcionar como uma saída viável para enfrentar dificuldades recorrentes e também excepcionais.

Este novo cenário tecnológico torna-se cada dia mais familiar à sociedade. Mas a escola, infelizmente, demora a utilizar as inovações em seus meios de ensino. Os avanços tecnológicos têm sido incorporados, primeiramente, nos setores mais industriais da sociedade, depois em domicílios e, por último, no ambiente escolar. (CAMPOS, F. et al.2003, p. 9)

O presente artigo, traz o relato de experiências dos alunos da Engenharia de Computação na condução do aprendizado da disciplina Desenho por meio de Ambiente Virtual de Aprendizagem(AVA) associado às tecnologias de modelagem 3D na execução das atividades proposta pela disciplina em 2020.

\section{FUNDAMENTAÇÃO TEÓRICA}

A Computação está presente nas mais diversas áreas de conhecimento e ensino, como importante ferramenta facilitadora do processo de ensino-aprendizagem. Tais modificações contribuem diretamente com o processo de criação de novas maneiras de ensinar e aprender (TIBÚRCIO, 2007).

Diante de numerosas inovações no meio profissional, observa-se na ensino em Desenho para Engenharia a preocupação quanto a adaptação da disciplina às novas requisições dos contratantes. Essa é necessária para que se possa assegurar o processo de aprendizagem do discente e prepará-lo para a aplicação do conhecimento teórico em uma nova conjuntura do mercado de trabalho (GOMES et al., 2016).

Conforme Bastos (2006) apud Berbel (2011) a metodologia ativa é um processo que visa estimular a aprendizagem e a curiosidade do estudante para pesquisar, refletir e analisar possíveis situações para tomada de decisão, sendo o professor apenas o facilitador desse processo.

A partir de uma maior interação do aluno no processo de construção do próprio conhecimento, que, conforme explicitado anteriormente, é a principal característica de uma abordagem por metodologias ativas de ensino, o aprendiz passa a ter mais controle e participação efetiva na sala de aula, já que exige dele ações e construções mentais variadas, tais como: leitura, pesquisa, comparação, observação, imaginação, obtenção e organização dos dados, elaboração e confirmação de hipóteses, classificação, interpretação, crítica, busca de suposições, construção de sínteses e aplicação de fatos e princípios a novas situações, planejamento de projetos e pesquisas, análise e tomadas de decisões (Souza; Iglesias; Pazin-Filho, 2014).

Conforme Bastos (2006) apud Berbel (2011) a metodologia ativa é um processo que visa estimular a aprendizagem e a curiosidade do estudante para pesquisar, refletir e analisar 
possíveis situações para tomada de decisão, sendo o professor apenas o facilitador desse processo

A partir de uma maior interação do aluno no processo de construção do próprio conhecimento, que, conforme explicitado anteriormente, é a principal característica de uma abordagem por metodologias ativas de ensino, o aprendiz passa a ter mais controle e participação efetiva na sala de aula, já que exige dele ações e construções mentais variadas, tais como: leitura, pesquisa, comparação, observação, imaginação, obtenção e organização dos dados, elaboração e confirmação de hipóteses, classificação, interpretação, crítica, busca de suposições, construção de sínteses e aplicação de fatos e princípios a novas situações, planejamento de projetos e pesquisas, análise e tomadas de decisões (Souza; Iglesias; Pazin-Filho, 2014).

Ademais, Aprendizagem colaborativa, caracterizada por Dillenbourg (1999) como uma situação de aprendizagem na qual duas ou mais pessoas aprendem ou tentam aprender algo juntas, não é algo novo. Também deve-se ter em vista as possibilidade intrínsecas a essa modalidade que, na perspectiva de Campos et al. (2003, p.26, apud CASTILHO et al., 2014, p. 54), "é uma técnica ou proposta pedagógica na qual estudantes ajudam-se no processo de aprendizagem, atuando como parceiros entre si e com o professor, com o objetivo de adquirir conhecimento sobre um dado objeto."

Nas palavras de Gerdy (1998, apud WIERSEMA, 2000, p. 3) “o aprendizado é aprimorado quando se parece mais com um esforço de equipe do que com uma corrida solo. [...] . Compartilhar as ideias de uma pessoa e responder às dos outros melhora o pensamento e aprofunda a compreensão."

Adicionado a isso, o fato do Isolamento social ocorrido no início do ano de 2020, tornou necessária a adequação dos métodos de ensino da disciplina de Desenho para Engenharia na Universidade Federal do Ceará, a fim de conciliar o desenvolvimento da capacidade de representação gráfica e a noção espacial do discente com a aquisição de conhecimentos tecnológicos de forma remota.

As tecnologias de AVA (Ambiente Virtual de Aprendizagem) somadas as específicas de computação gráficas, estão alterando as formas de desenvolvimento de competências, e cada vez mais presente nas escolas, como importante ferramenta facilitadora do processo de ensino- aprendizagem. Tais modificações influenciam de forma direta o processo de criação de novas maneiras de ensinar e aprender, conforme Tibúrcio, (2007) é essencial a utilização de metodologias diferenciadas, como a utilização de softwares de fácil manuseio, atividades em grupo e a elaboração de projetos com objetos conhecidos pelos alunos.

Com tais metodologias é esperado o maior engajamento por parte dos alunos. Para demonstrar as vantagens da utilização das metodologias citadas acima, ainda que executada em meio ao isolamento social, o presente artigo mostra a partir do relato de caso do projeto de modelagem 3D, perpassando as vantagens e as dificuldades no processo de aprendizagem, obtendo como resultado uma maneira mais engajadora de ensinar os futuros alunos da mesma disciplina, concluindo, dessa maneira, que o melhor modo para se desenvolver o aprendizado de desenho técnico é por meio de uma maior interação com os objetos que os alunos têm mais afinidade. 


\subsection{Tecnologias de Aprendizagem virtuais}

Um grupo virtual é constituído, segundo Lévy (2000, p. 127, apud SCHERER; BRITO, 2014 , p. 55), "sobre projetos mútuos, em um processo de cooperação ou de troca, tudo isso independentemente das proximidades geográficas e das filiações institucionais." Se trata de um curso de reciprocidades e compartilhamentos de aprendizados que ultrapassa barreiras geográficas e permite a construção do conhecimento. Nessa perspectiva, as ferramentas digitais são as alternativas adotadas para manutenção e estímulo de colaboração entre os participantes. Não deve se perder de perspectiva que os atores principais continuam a ser os participantes de modo que os meios virtuais "dependem da atitude destes frente às propostas de ações nos ambientes virtuais de aprendizagem.” (SCHERER; BRITO, 2014, p.54).

Além disso, devem existir mecanismos que visem a continuidade das atividades pois se tratam de realidades e disposições diferentes. O papel do tutor deixa de ser o principal e passa a ser o de coadjuvante, um coadjuvante sempre atento que acompanha e pontua quando necessário, visando sempre garantir aprendizagem e continuidade do processo. $\mathrm{Na}$ modalidade de ensino a distância o professor funciona, de acordo com Oliveira e Silveira (2014, p.97-98, apud NASCIMENTO; SILVA, 2018, p. 77), "como um colaborador ou um intermediador do aprendizado, que deve monitorar e acompanhar o estudante durante todo o processo, até que este consiga se tornar mais proativo e autônomo [...].”

Para facilitar a interação entre os participantes em ambientes virtuais são utilizadas diversas ferramentas. Essas ferramentas podem ser divididas de maneira mais ampla em ferramentas síncronas e assíncronas. Onde as síncronas compreendem aquelas cuja proposta é a comunicação em tempo real, ou seja, membros conectados ao mesmo tempo. As ferramentas assíncronas, contrariamente, dizem respeito às que não requerem interação em tempo real. Nascimento e Silva (2018) com base em pesquisa bibliográfica levantaram ferramentas utilizadas nestes ambientes, sendo estas: material didático-pedagógico; diário; wiki; chat; portfólio.

A plataforma Classroom da Google é um Ambiente virtual de ensino, onde o conteúdo e compartilhado, fórum são criados, possibilitando os alunos discutirem e interagirem com o professor e entre si. Essas características estão presentes na Figura 1.

Figura 1 - Ambiente de Aprendizagem Virtual da disciplina DPE

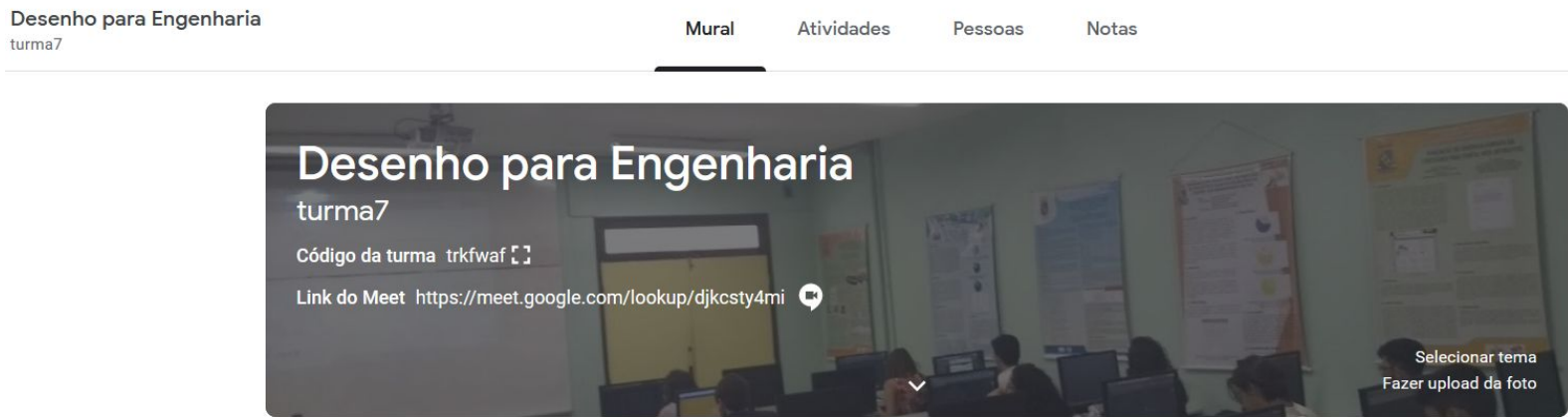

Fonte: Elaborado pelos autores <https://classroom.google.com/u/0/c/ODM1ODYwMzg5MTha $>$ 


\section{METODOLOGIA}

A organização utilizada no grupo de estudos se deu da seguinte forma: inicialmente foi feito um planejamento sobre o que deveria ser estudado além do levantamento de materiais necessários para a execução do trabalho

As reuniões foram feitas pelo MEET GOOGLE e os desenhos puderam ser desenvolvidos com o software de modelagem $3 \mathrm{~d}$ da preferência do grupo, dentro de um cronograma em formato de roteiro (ver Figura 2)

Figura 2 - Webconferência no MEET Google

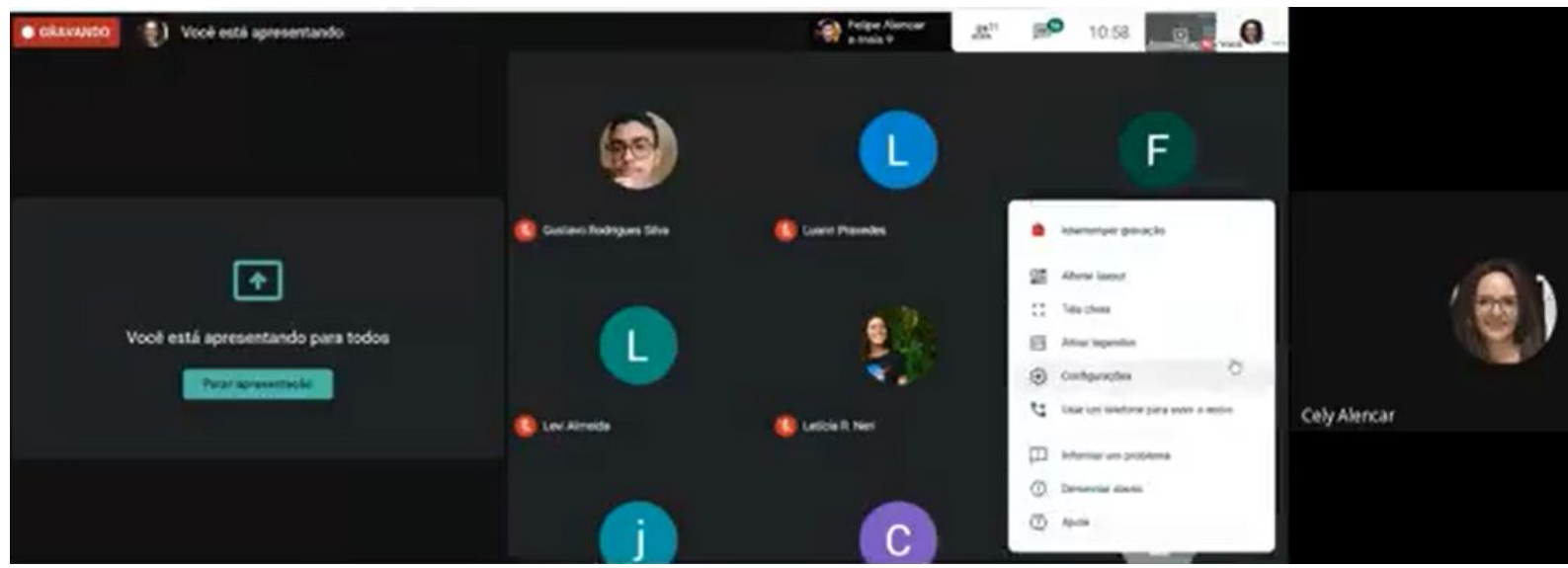

Fonte: Elaborado pelos autores $<\underline{\text { https://meet.google.com/lookup/djkcsty4mi }>}$

Em seguida, começou-se a se colocar em prática o que foi planejado, assim, cada integrante estudava e colocava o assunto em prática. As dúvidas que surgiam eram compartilhadas em grupo de mensagem instantânea (WhatsApp), também por meio de reuniões semanais nas quais era relatado o progresso do grupo e o compartilhamento dos conhecimentos.

O ambiente de comunicação do grupo foi majoritariamente por aplicativo de mensagens (WhatsApp) e por aplicativo de chamadas de voz e vídeo (Discord), de modo que cada parte da atividade possuía um responsável, também contaram com uma pasta virtual (google drive) com os desenhos desenvolvidos, material de estudo inicial e um arquivo escrito construído pelos membros. Cabe ressaltar que todos tinham a oportunidade de incluir novos arquivos e editar os existentes, de fato eram encorajados. A pasta também funcionava como meio para acompanhamento macro do grupo, visto que permitia verificar atualizações.

\section{RESULTADOS E DISCUSSÃO}

Quase a totalidade do conhecimento utilizado para a execução do projeto de modelagem do Playstation 4 (PS4) foi adquirido através de encontros, tanto presenciais quanto virtuais, 
com professor da Disciplina. Metodologias $\operatorname{passivas}^{1}$ e $\operatorname{ativas}^{2}$ foram utilizadas pela docente para transmitir os conhecimentos necessários tanto para a aprovação na disciplina quanto para a atuação dos discentes no futuro mercado de trabalho.

\subsection{Confecção das vistas ortográficas e cavaleira}

As geometrias geradas no trabalho em CAD seguem a sequência lógica para a construção de peças e baseia-se na escolha de um plano para criar o esboço $2 \mathrm{D}$, na sequência a forma geométrica principal da peça que se representa com entidades de esboço disponíveis. No sistema CAD se pode criar com geometria 3D com precisão (GD) (Figura 2) Dois dos três alunos integrantes do grupo foram os responsáveis da execução das vistas ortográficas (Figura 1) e cavaleira (Figura 3), a importância destas vistas se deve ao compreendimento da geometria do objeto que futuramente viria a ser modelado no Sketchup. Para tanto, as medidas puderam ser obtidas por meio de um dos integrantes do grupo que possuía o objeto que seria modelado, no caso o Playstation 4 (PS4), com as medidas em mãos, os alunos realizaram a confecção à mão livre, pois era requisitado pela docente que fossem executadas de tal maneira.

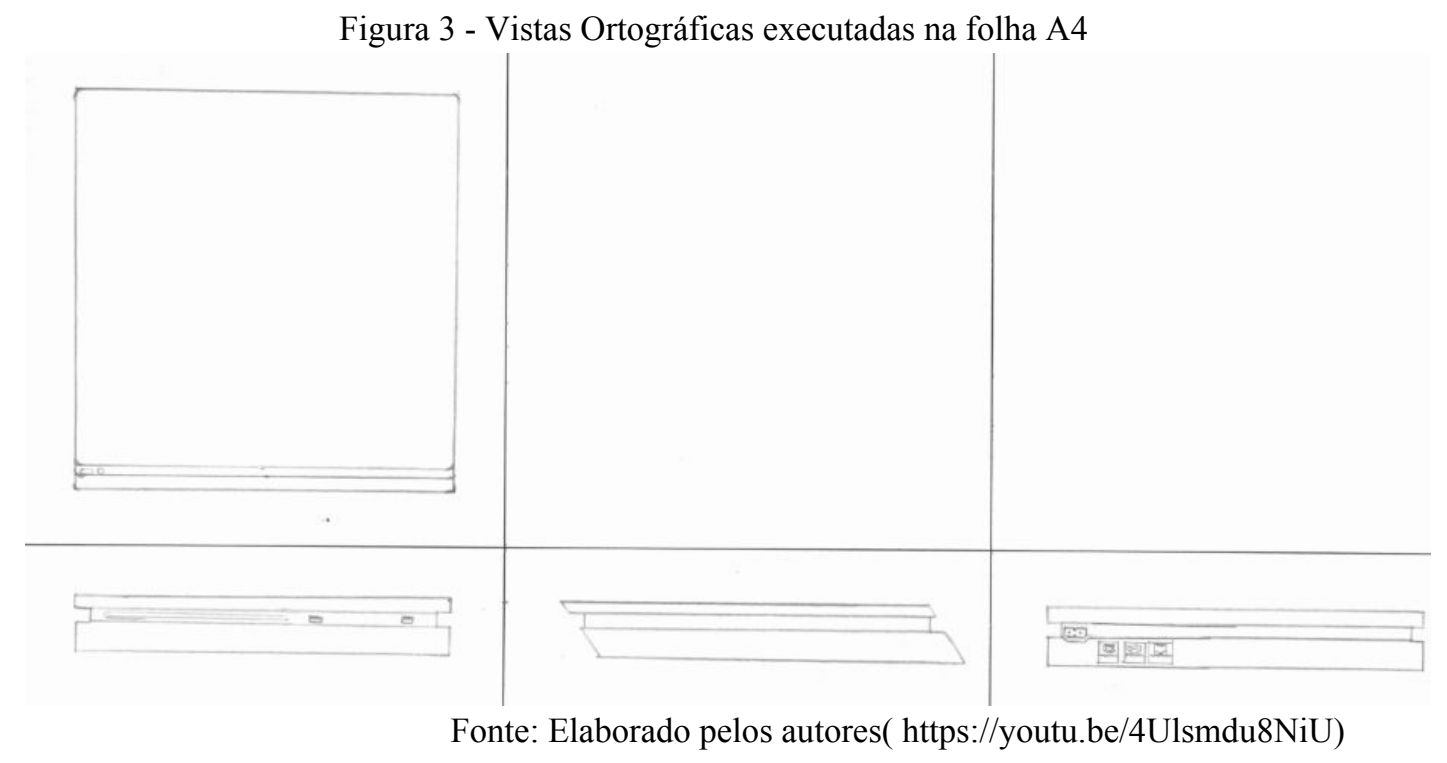

Figura 4 - Vista Cavaleira executada na folha A3

\footnotetext{
${ }^{1}$ Onde o protagonista é o professor, é baseada em trabalhos individuais, desenhos manuais e atividades com sólidos abstratos e geométricos.

${ }^{2}$ Onde o protagonista é o aluno, é baseada em trabalhos em grupo, desenhos digitais, por meio de softwares $C A D$, e atividades relacionadas ao curso.
} 


\section{(1) da $A B E N G E$}

\section{"Os desafios para formar hoje o engenheiro do amanhã"}

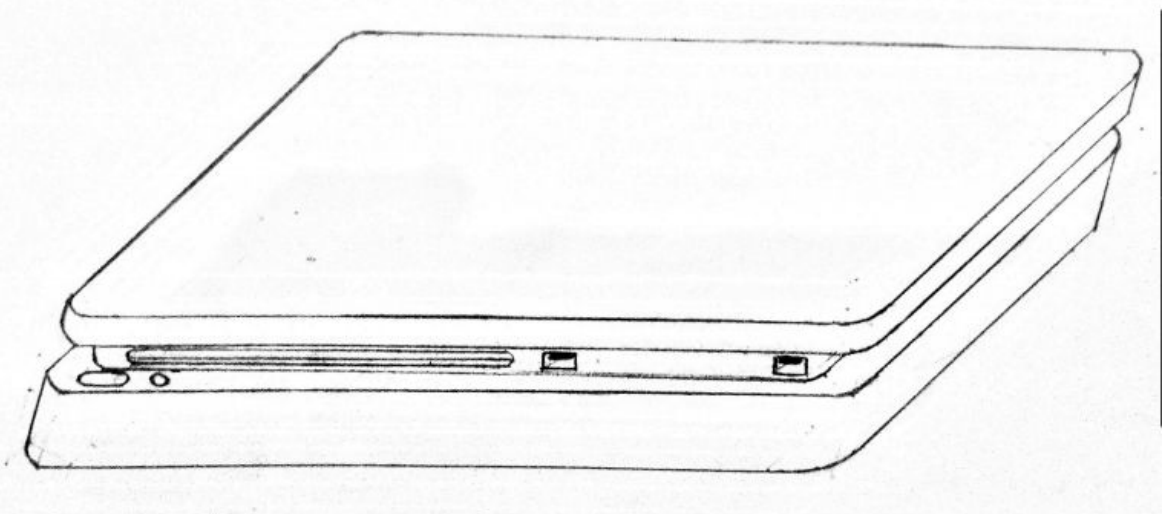

Fonte: Elaborado pelos autores

\subsection{Execução do sólido no Sketchup}

O terceiro integrante ficou responsável pela execução do sólido no Sketchup, a escolha desse software se deu pela dificuldade de adaptação ao AutoCAD 3D, a execução do modelo demorou 3 semanas após o início da execução no Sketchup, já que a equipe já havia tentado a execução no AutoCAD 3D, sem êxito, e, durante essas, a cada modificação no sólido a equipe se reunia para conferir a execução, ao final de 4 dias o sólido estava pronto e a equipe estava pronta para a apresentação do trabalho.

Após um minicurso de Sketchup executado em um dos encontros da disciplina, a equipe se mostrou favorável à execução do projeto no Sketchup por parecer mais acessível e fácil de manusear. Dado o início da execução, o aluno teve dificuldades inicialmente para se adaptar a ferramenta, mas ao fim do $1^{\text {a }}$ dia já tinha se adaptado à ferramenta e sabia utilizar uma vasta quantidade de comandos. No $2^{\circ}$ dia, o sólido já ganhava molde, a parte inferior e superior já haviam sido feitas, faltando somente a parte central pois continha maior parte dos detalhes, curvas e entradas do próprio console. No $3^{\circ}$ dia, após consulta em vídeos no YouTube, a parte central encontrava-se praticamente concluída, faltando somente as entradas. No $4^{\circ}$ dia $o$ sólido havia sido concluído, como mostrado na Figura 5 com os detalhes visuais e logomarca em cima do console e os botões na Vista Frontal. 
Figura 5 - Vista em perspectiva das 3 partes (inferior,central e superior) do Playstation 4 (PS4)

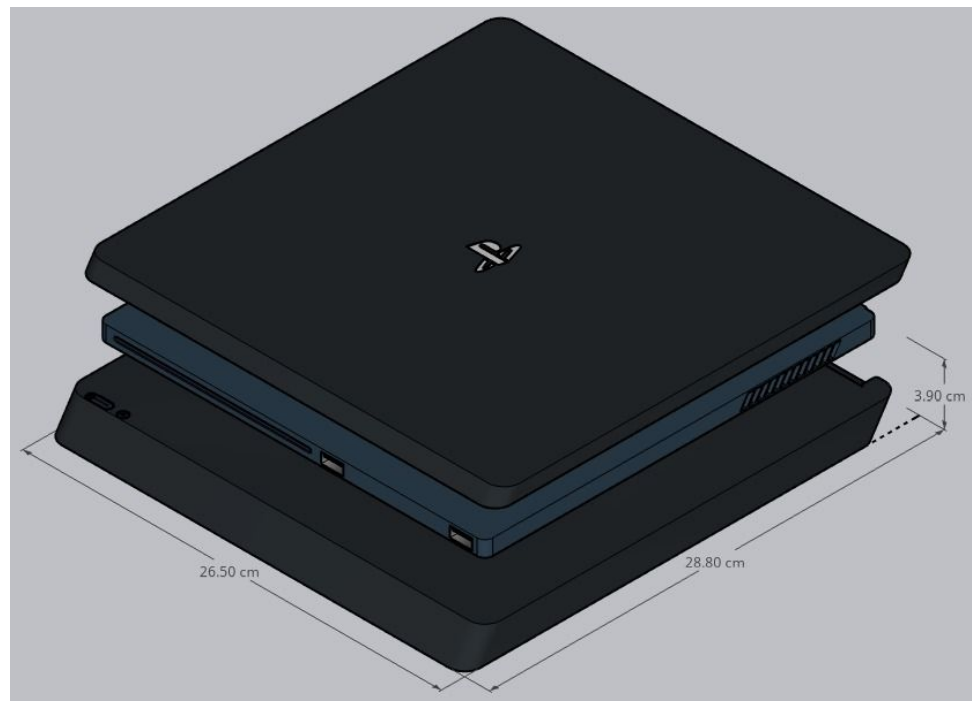

Fonte: Elaborado pelos autores

\section{CONSIDERAÇÕES FINAIS}

O objeto foi executado de forma satisfatória, apresentou todos os aspectos avaliados na disciplina de Desenho para Engenharia e pode servir de modelo para futuras turmas ingressantes nos cursos de Engenharia.

Pode-se concluir que a utilização de metodologias ativas, principalmente em período de ensino remoto, pode favorecer a integração entre os discentes e a melhor aplicação dos conhecimentos obtidos por meio dos encontros semanais, sejam esses presenciais ou remotos.

Também pode-se afirmar que a liberdade dada aos discentes para que escolhessem o objeto a ser representado foi de grande importância, pois esses puderam escolher objetos conhecidos e desse modo sentiram-se mais engajados para executar tal tarefa.

O estudo teve como objetivo principal apresentar aprendizagem desenvolvida de forma a retomar todos os conhecimentos adquiridos no desenvolver da disciplina e analisar a qualidade dos projetos desenvolvidos.

\section{Agradecimentos}

Nosso agradecimento a professora da Disciplina de Desenho para Engenharia da Universidade Federal do Ceará e ao Departamento de Integração Acadêmica e Tecnológica (DIATEC), da Universidade Federal do Ceará.

\section{REFERÊNCIAS}

BERBEL, Neusi Aparecida Navas. As metodologias ativas e a promoção da autonomia de estudantes. Disponível em $<$ http://www.uel.br/revistas/uel/index.php/seminasoc/article/view/10326/10999> Acesso em 23/09/2020 às 21:39. 
SCHERER, S.; BRITO, G. da S. Educação a distância: possibilidades e desafios para a aprendizagem cooperativa em ambientes virtuais de aprendizagem. Educar em Revista, Curitiba, Brasil, Edição Especial n. 4/2014, p. 53-77. Editora UFPR

CASTILHO, Antonio M. Dias et al. A rede social facebook como ferramenta pedagógica no processo de ensino-aprendizagem de língua inglesa. Revista Transformar, Itaperuna, n. 6, p. 42-63, 2014.

CLEBER, R. A importância do 3D na formação de novos engenheiros. Disponível em: https://www.plataformacad.com/a-importancia-do-3d-para-novos-engenheiros/. Acesso em 20 jul. 2020.

DILLENBOURG, Pierre. What do you mean by collaborative learning?. In: DILLENBOURG, Pierre (Ed.). Collaborative-learning: Cognitive and Computational Approaches. $1^{\text {a }}$. ed. Oxford: Elsevier Publishing, 1999. p. 1-19.

GOMES, William J.; LOPES, Celi E. Ensino de desenho técnico no curso de edificações. In: Encontro nacional de educação matemática 2016: Educação Matemática na Contemporaneidade: desafios e possibilidades, Anais..2016, São Paulo.

LÉVY, Pierre. Cibercultura. Tradução de Carlos Irineu da Costa. 2. ed. São Paulo: Ed. 34, 2000. 264p.

MONNERAT, Lúcia Patrícia et al. Uma abordagem para a melhoria do processo de ensino-aprendizagem em desenho técnico utilizando métodos e técnicas da computação. 2012. Dissertação (Mestrado em Ciência da Computação) Universidade Federal de Viçosa. Viçosa, MG, p. 171. 2012.

NASCIMENTO, Francisco Elionardo de Melo; SILVA, Denilson Gomes. Educação Mediada por Tecnologia: inovações no processo de ensino e aprendizagem - uma revisão integrativa. Abakós, Belo Horizonte, v. 6, ed. 2, p. 72-91, maio 2018.

OLIVEIRA, Carolina Dias de; SILVEIRA, Ricardo Azambuja. Avaliação do uso das ferramentas tecnológicas em ambientes virtuais de ensino e aprendizagem em educação a distância: Um estudo de caso do e-tec/cefet-mg. Anais do ESUD2014, XI Congresso Barasileiro de Ensino Superior a Distância Florianópolis/SC, 2014. Disponível em:

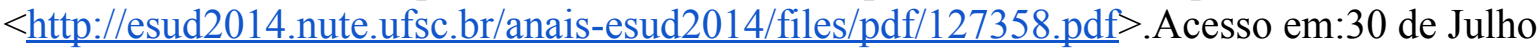
de 2020 .

TIBÚRCIO, T. M. S. The Impact of High-tech learning environment on Pupils' Interaction. PhD Thesis. The University of Reading. Reading-UK, 2007. 
SANTOS, Jairo Campos dos. A Informática na Educação contribuindo para o processo de revitalização escolar. V CINFE - CONGRESSO INTERNACIONAL DE FILOSOFIA E EDUCAÇÃO, p. 1-16, maio 2010.

SOUZA, Cacilda da Silva; IGLESIAS, Alessandro Giraldes; PAZIN-FILHO, Antonio. Estratégias inovadoras para métodos de ensino tradicionais - aspectos gerais. Medicina, v. 47, n. 3 , p. $284-292,2014$.

WIERSEMA, N. How does Collaborative Learning actually work in a classroom and how do students react to it? A Brief Reflection. Disponível em:

https://eric.ed.gov/?id=ED464510. Acessado em 30 de julho de 2020.

\title{
GRAPHIC COMPUTING TECHNOLOGIES AND VIRTUAL LEARNING TO ENABLE REMOTE DESIGN PRACTICES FOR ENGINEERING
}

\begin{abstract}
This article shows the report of learning remotely in the discipline of Design for Engineering of the Federal University of Ceará, by students of Computer Engineering. With the social isolation caused by Covid-19, there was a need to carry out Teaching and activities remotely. Thus, the methodology used was the use of web conferencing technologies for meetings and computer graphics in the construction of drawings. This case report describes all the steps performed by the students, mediated by computer graphics technologies in the modeling of pieces with techniques of Geometric Design. The solid executed was Playstation 4 (PS4) due to the presence of such an object in the daily lives of the members of the group. The results obtained were satisfactory and the experiences acquired, such as individual responsibility, group work and collaboration, were observed in the assessed skills.
\end{abstract}

Keywords: Learning, Remote teaching, Engineering design, Computer graphics. 